\title{
SMOOTH SWITCHING OF MICRO GRID OPERATION MODE OF VIRTUAL SYNCHRONOUS GENERATOR
}

\author{
Gao Jirong ${ }^{1}$, Tao Wang ${ }^{1}$, Shubing Yan ${ }^{1}$, Haroon Rashid ${ }^{2}$ \\ ${ }^{1}$ Shandong Polytechnic College, Shandong, China \\ 2Department of Structures \& Environmental Engineering, University of Agriculture 38000 Faisalabad, Pakistan \\ Email: guojirong00053@126.com
}

\begin{abstract}
In this study, the smooth switching of the control strategy of the micro grid inverter is premeditated to improve the stability of the grid operation. Virtual synchronous generator control and constant power control are combined to form a dual-mode parallel inverter control strategy. The pre-synchronization control strategy is designed for the switching process from island to grid. At the same time, the traditional state following controller is improved for the switching process from grid connection to island operation. Simulation of microgrid operation mode switching is achieved. The voltage and current of the micro grid bus are very stable, basically no fluctuation, no matter at the time of $0.2 \mathrm{~s}$ start-up pre-synchronization control or $0.3881 \mathrm{~s}$ pre-synchronization end grid connection. The voltage and power impact of the micro grid under the traditional state following control is significantly higher than that of the improved state following control. In this study, each control strategy not only realizes the stable operation of the microgrid in island mode and grid connected mode, but also realizes the smooth transition in the mode switching process, which proves its effectiveness.
\end{abstract}

Keywords: Virtual Synchronous Generator, Microgrid, Smooth Switching, Constant Power Control, Inverter.

\section{Introduction}

As a new generation technology, distributed generation technology has many advantages over traditional generation methods. However, due to the randomness of renewable energy such as solar energy and wind energy, when the proportion of its access to the power grid gradually higher and higher, the problems in the system also ascended. Generally, the difficulty of power dispatching, peak load regulation, frequency modulation and power grid capacity planning will increase simultaneously. At the same time, it will damage the power quality of the power grid and even threaten the safe and stable operation of the power grid, to a large extent causes distributed generation limitations of technological development [1]. In order to solve these problems effectively, researchers all over the world have put forward many solutions. Among them, the concept of micro grid proposed by a research association of the United States has aroused more discussion in the academic community. With the deepening of research, microgrid, as an efficient form of distributed power generation, has become a research hotspot and development trend in the current power industry [2]. In microgrid, grid connected inverter, as an important interface between distributed power and microgrid, must be equipped with reasonable control strategy to keep the microgrid running smoothly. However, although the traditional inverter control strategy can meet the requirements of distributed power supply under various operation modes of microgrid. Meanwhile, it cannot provide the necessary inertia and damping for the system, thus affecting the stability of microgrid operation [3-4].

As a new inverter control strategy in recent years, a virtual synchronous generator can effectively make up for the disadvantages of traditional inverter control strategy and realize the necessity of micro grid control and smooth switching. Some scholars have proposed a frequency difference with a free smooth switching control strategy based on virtual synchronous generator.

Based on traditional droop control, a voltage amplitude feedback control loop and a fuzzy PID (proportional integral differential controller) controller are added to realize the no difference tracking of the pre-set voltage, effectively reducing the power oscillation and bus voltage deviation during the operation mode switching of microgrid [5]. From the perspective of research results, there are still some aspects to be improved. After all, microgrid is still a new thing, and the research of 
various countries is also in the stage of theoretical exploration and demonstration projects.

Therefore, in this study, the dual-mode parallel inverter control strategy combining virtual synchronous generator control and constant power control is studied. Moreover, a pre-synchronization control strategy is designed in addition to phaselocked loop technology, and the traditional state following controller is improved. The simulation results show that the control strategies of this study not only realize the stable operation of microgrid in island mode and grid mode, but also realize the smooth transition in the mode switching process.

\section{Method}

\subsection{Smooth switching control of microgrid based on virtual synchronous generator}

When connected to the grid, the voltage of the micro grid system is clamped by the large grid. It does not need to support the internal voltage of the system itself. However, it only needs to control the distributed power supply to keep constant power to transmit active power and reactive power to the system [6]. Therefore, when the micro grid is connected to the grid, the simpler and more mature PQ (constant power) control strategy can be used to regulate the grid connected inverter of the distributed power supply.
So that the micro source can output power to the system according to the given active power and reactive power reference value.

When the micro grid operates in isolated island, the voltage and frequency of the system will lose the support of the large grid. At this time, it is necessary to collect the voltage and current information of the micro grid common bus in real time. Furthermore, feed it back to the control strategy to adjust the output value of the grid connected inverter, so that the voltage and frequency in the micro grid system can continue to maintain stable operation at the rated value [7-8]. Therefore, in order to meet the above conditions, a new virtual synchronous generator control strategy can be used to control the grid connected inverter when the micro grid is in island operation. Using the distributed generation of a new control strategy can not only adjust the voltage and frequency of the micro grid bus through the output, but it also imitates the characteristics of the synchronous generator. This ultimately provides certain inertia and damping for the micro grid system and improves the stability of the micro grid operation. Besides, when the microgrid needs to be connected to the power grid, it can also follow the principle of synchronous device of synchronous generator to realize the stable switching of microgrid from island to grid operation mode.

The specific control structure is shown in Figure 1:

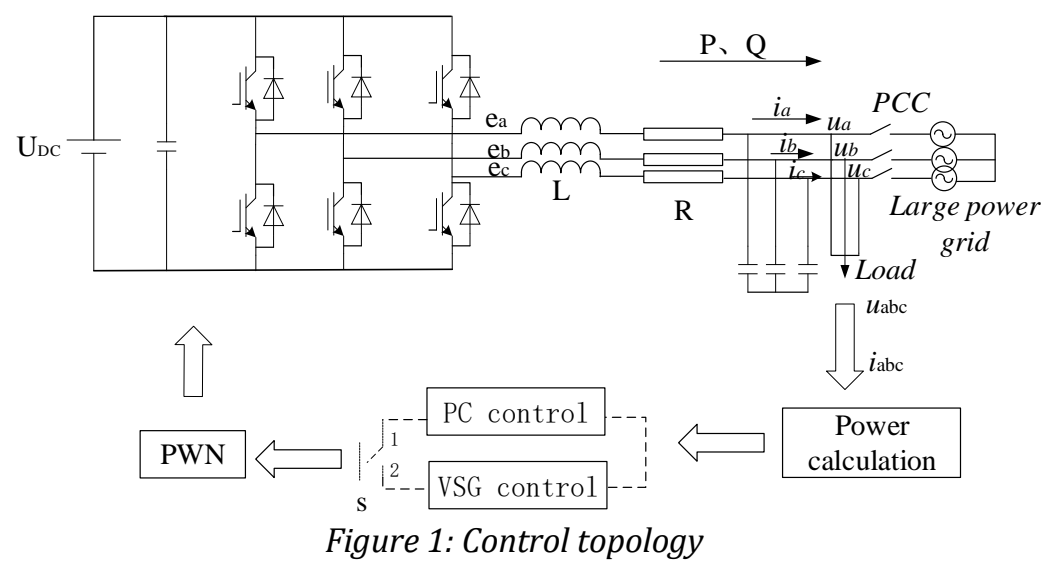

When the micro grid is in stable operation, the grid connected inverter is only affected by one control strategy. Among them, PQ control is suitable for grid connected operation mode, VSG control (virtual synchronous generator control) is suitable for island operation mode. For example, when the microgrid is in island operation mode, both control strategies are in operation, only the output of VSG control acts on the grid connected inverter. In contrast, the output of PQ control does not affect the inverter. When the grid connection signal is received and the PCC switch is closed to complete the grid connection action, the switch $\mathrm{S}$ moves from position 2 to position 1 , the output result of PQ control starts to control the grid connected inverter, and the output result of VSG control is vacant. Of course, this is only the control mode when the micro grid is in steady state. In order to realize smooth switching between operation modes and eliminate the voltage and current impact caused by the instant of connection and disconnection as well as switching $\mathrm{S}$ action, further design of control strategy is needed.

\subsection{Phase-locked loop (PLL) technology}

The basic function of PLL is to track and lock the phase of AC signal, and extract the frequency and amplitude information of related signal when 
necessary. PLL technology is a vital part of the control strategy of grid connected inverter, and it is also the key to the normal and effective operation of the control strategy and the realization of its functions. The accuracy and precision of phase tracking greatly affect the performance of grid connected inverter. If the phase-locked is not accurate or the accuracy is not high enough, it will seriously affect the quality of the output power of the inverter, and even damage the electrical equipment under the jurisdiction of the microgrid system [9-10]. In the grid connected inverter, the d-q Synchronous Reference Frame PLL (SRF-PLL) is often used to collect the voltage amplitude, phase, frequency and other information. The control structure is shown in Figure 2:

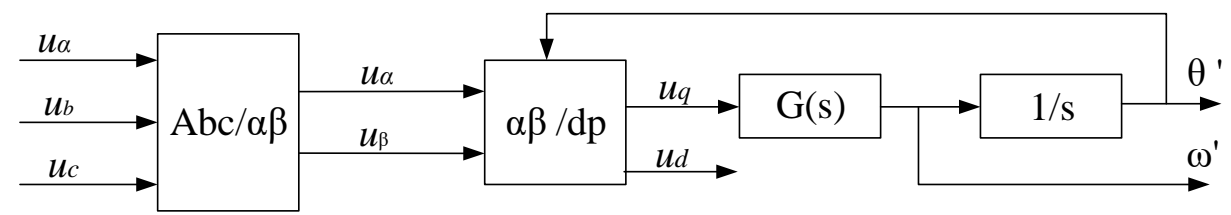

Figure 2: SRF-PLL control structure

The three-phase voltage input is expressed as:

$$
\left\{\begin{array}{c}
u_{a}=U_{m} \cos \omega t \\
u_{b}=U_{m} \cos \left(\omega t-120^{\circ}\right) \\
u_{c}=U_{m} \cos \left(\omega t+120^{\circ}\right)
\end{array}\right.
$$

Clark and Park transform the three-phase voltage.

The AC quantity in $\mathrm{ABC}$ three-phase static coordinate system is changed into DC quantity in d-q synchronous rotating coordinate system.

The transformation equations are as follows:

$$
\begin{aligned}
& {\left[\begin{array}{l}
u_{\alpha} \\
u_{\beta}
\end{array}\right]=\frac{2}{3}\left[\begin{array}{rrr}
1 & -\frac{1}{2} & -\frac{1}{2} \\
0 & \frac{\sqrt{3}}{2} & -\frac{\sqrt{3}}{2}
\end{array}\right]\left[\begin{array}{l}
u_{a} \\
u_{b} \\
u_{c}
\end{array}\right]=U_{m}\left[\begin{array}{l}
\cos \omega t \\
\sin \omega t
\end{array}\right]=U_{m}\left[\begin{array}{l}
\cos \theta \\
\sin \theta
\end{array}\right]} \\
& {\left[\begin{array}{l}
u_{d} \\
u_{q}
\end{array}\right]=\left[\begin{array}{cc}
\cos \theta^{\prime} & \sin \theta^{\prime} \\
-\sin \theta^{\prime} & \cos \theta^{\prime}
\end{array}\right]\left[\begin{array}{l}
u_{\alpha} \\
u_{\beta}
\end{array}\right]=U_{m}\left[\begin{array}{l}
\cos \left(\theta-\theta^{\prime}\right) \\
\sin \left(\theta-\theta^{\prime}\right)
\end{array}\right]}
\end{aligned}
$$

In the above equation: $\theta$ is the angle of input voltage; $\theta^{\prime}$ is the estimated angle of PLL output. It can be seen from equation (3) that in order to ensure that the output angle of the PLL matches the angle of the input voltage accurately, it is necessary to ensure that $u_{q}=0$. The control block diagram can be obtained by simplifying the control structure diagram in Figure 2, as shown in Figure 3 below:

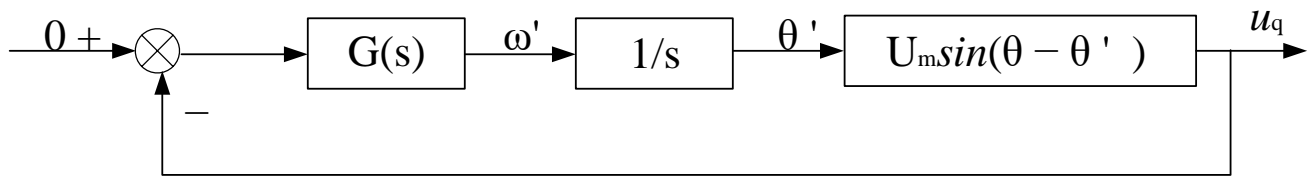

Figure 3: Simplified SRF-PLL control structure

However, due to the non-linear part $\sin \left(\theta-\theta^{\prime}\right)$ in the block diagram of Figure 3 above, it is challenging to analyze and calculate the parameters of the PLL according to the control block diagram. Therefore, it is significant to linearize the control block diagram. Generally, when the phase-locked loop control enters the steady state, if there is $\theta^{\prime} \approx \theta$, that is, $\theta-\theta^{\prime}$ $=0$, then $\sin \left(\theta-\theta^{\prime}\right) \approx \theta-\theta^{\prime}$.

Therefore, after the linearization analysis of Figure 3 based on the above reasoning, the linearization model as shown in Figure 4 below can be obtained:

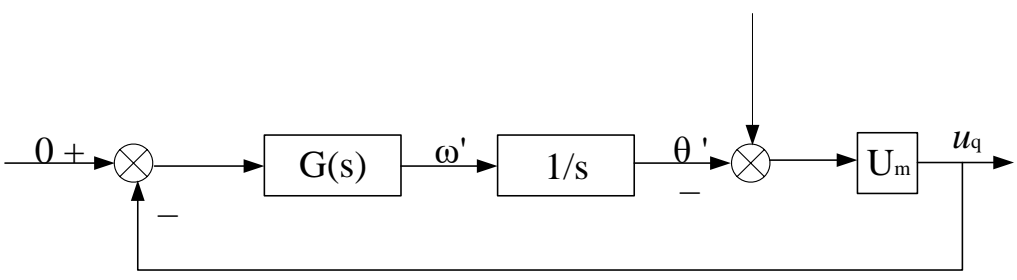

Figure 4: SRF-PLL linear block diagram 
It can be seen from the figure above that the PLL ensures that the output angle $\theta^{\prime}$ can accurately track the reference angle $\theta$ through the closed-loop control, so as to realize the function of phase-locked.

\subsection{Switch from island to grid mode}

Micro grid should not only ensure the stability of steady-state operation but also ensure the smooth implicit transition of voltage and current when the operation mode is switched. When the micro grid is in island operation mode, the large grid is in different operation environment, and their control modes are not the same. During operation, the control strategy of grid connected inverter may adjust the amplitude and phase of output voltage according to the needs of microgrid. With the accumulation of time, there will be a specific deviation in the voltage phase between the microgrid and the large grid [11].
At this time, if some measures are not taken and the grid is connected rashly, the voltage waveform will be deformed and the power quality will be worsened. In severe cases, too much impulse current may be generated at the moment of connecting to the grid, failing in connecting to the grid.

Phase $\mathrm{A}$ is taken as an example. In islanding operation mode, the terminal voltage $u_{a}$ and grid voltage $u_{g a}$ of virtual synchronous generator are respectively expressed as follows:

$$
\begin{aligned}
& u_{g a}=U_{1} \cos \left(\omega_{0} t+\theta_{1}\right) \\
& u_{a}=U_{2} \cos \left(\omega t+\theta_{2}\right)
\end{aligned}
$$

In general, even if the microgrid operates in an isolated island, it can be considered that the difference between its voltage amplitude and the grid voltage amplitude is not significant, so it can be considered as follows:

$$
U_{1} \approx U_{2}=U
$$

Then, the instantaneous difference $\Delta u$ between $u_{a}$ and $u_{g a}$ is:

$$
\mathrm{V} u=u_{g a}-u_{a}=2 U \sin \left(\frac{\omega_{0}-\omega}{2}+\frac{\theta_{1}-\theta_{2}}{2}\right) \cos \left(\frac{\omega_{0}+\omega}{2}+\frac{\theta_{1}+\theta_{2}}{2}\right)
$$

It can be seen from the above equation that there may be a voltage phase deviation between the micro grid and the large grid operating in an isolated island. Moreover, when the deviation is maximum, the difference of in-phase voltage may reach $2 \mathrm{U}$. Therefore, when the fault of the massive power grid is repaired, or the micro power grid needs to be incorporated into the large power grid according to the actual situation, the pre-synchronization control strategy must be adopted to control the voltage phase of the micro power grid to keep consistent with the voltage phase of the large power grid, to reduce the voltage and current impact at the moment of switching from island to grid connection.

To sum up, the key to realizing the smooth switching from islanding to grid connected operation mode lies in how to solve the problem that the voltage amplitude and phase at both ends of the grid connected switch PCC keep synchronous with the large grid. According to the requirements, the presynchronization control unit can be divided into amplitude pre-synchronization and phase presynchronization. The controller superimposes the amplitude pre-synchronization signal and phase presynchronization signal to $\mathrm{E}_{0}$ and $\theta_{0}$ link, respectively. Through the adjustment of $E$ and $\theta$, the synchronization of the two is realized. The control principle is as follows:

$$
\begin{aligned}
& E=E_{0}+\Delta E=E_{0}+\left(K_{p E}+\frac{K_{i E}}{S}\right)\left(E_{g}-E\right) \\
& \theta=\theta_{0}+\Delta \theta=\theta_{0}+\left(K_{p \theta}+\frac{K_{i \theta}}{S}\right)\left(\theta_{g}-\theta\right)
\end{aligned}
$$

In the equation: $\Delta E$ and $\Delta \theta$ are pre-synchronous signals of amplitude and phase respectively;

$E_{g}$ and $\theta_{g}$ are voltage amplitude and phase of large power grid respectively;

$E$ and $\theta$ are voltage amplitude and phase of microgrid respectively;

$\mathrm{K}_{\mathrm{pE}}, \mathrm{K}_{\mathrm{iE}}, \mathrm{K}_{\mathrm{p} \theta}$ and $\mathrm{K}_{\mathrm{i} \theta}$ are the proportion, integration and coefficient of amplitude and phase respectively.

When the micro grid needs to be connected to the grid and send the signal to the pre-synchronization control unit, switches S1 and S2 are closed, and the pre-synchronization signal is sent to the corresponding controller respectively. After the amplitude and phase synchronization between the micro grid and the large grid, the presynchronization control process is completed, and the switches S1 and S2 are cut off to exit the presynchronization control. At the same time, the grid connected switch PCC is closed to complete the switch from island to grid mode.

\subsection{Switching from grid connection to island mode}

For microgrid, in order to improve the utilization rate of distributed power and the reliability of microgrid load, the microgrid is usually connected to the grid. However, it is also necessary to consider the situation of microgrid off grid operation due to failure or planning factors. At this time, it is not only necessary to disconnect the PCC switch between 
microgrid and large grid, but also to switch the control strategy of grid connected inverter in microgrid. Because the algorithm and purpose of the two control strategies are not the same, the output results are necessarily different. At this time, if any measures are not taken and the grid is disconnected abruptly, the microgrid will lose the support of the large grid to its voltage and frequency at the moment of leaving the grid. Although the virtual synchronous generator control strategy can control the inverter to quickly establish and stabilize the voltage and frequency of the microgrid, the difference of the control signal at the moment of control strategy switching will cause certain voltage and current impact at this moment. If not restrained, it will bring serious harm to the load in microgrid [12-13].

For microgrid off grid, in addition to planned off grid, it is more likely to be unplanned sudden off grid and unpredictable, so it is impossible to design control strategy in advance like an island to grid process to make the switching process smoother [14-15]. The key to solving the problem of excessive voltage and current impact at the moment of off grid is how to design a control strategy to minimize the impact on the voltage and current of microgrid when the controller is switched. In view of this, some researchers have proposed a state following controller, but its effect in reducing the switching transient voltage and current impulse is limited. In view of the defects of traditional state following controller, its working mechanism is further studied and analyzed, and its defects are improved. A new state following controller is proposed, as shown in Figure 5:

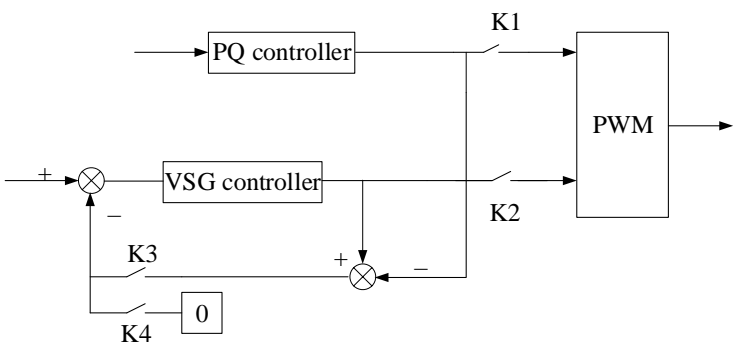

Figure 5: Improved state following controller

When the microgrid is connected to the grid, switches $\mathrm{K} 1$ and $\mathrm{K} 3$ are closed, $\mathrm{K} 2$ and $\mathrm{K} 4$ are disconnected, and $\mathrm{PQ}$ controller controls the grid connected inverter. At the same time, the difference between the output results of PQ controller and VSG controller is added to the input of VSG controller as negative feedback, which keeps the output results of VSG controller tracking the results of PQ controller, but does not affect the inverter. When the off-grid action occurs, the grid switch PCC is turned on. At this time, $\mathrm{K} 1$ is open, $\mathrm{K} 2$ is closed, $\mathrm{K} 3$ is still closed, $\mathrm{K} 4$ is open, and the output results of VSG controller are kept tracking the results of $\mathrm{PQ}$ controller, so as to avoid large amplitude oscillation of voltage and current of microgrid caused by different output results of different controllers at the moment of control strategy switching. Until the signal detector detects that the difference between the output results of PQ controller and the VSG controller is at the minimum value (the ideal state is 0 ), it is necessary to cut off K3 and close K4. VSG controller collects voltage and current signals of microgrid separately and acts on grid connected inverter, so PQ controller is no longer tracked. The result of $\mathrm{PQ}$ controller is in the vacancy state.

To a certain extent, the improved state following controller makes up for the defects of the traditional state following controller. It keeps the state following process until the difference between the output results of the two controllers is the minimum. At this time, the connection between the two controllers is cut off, and the VSG controller controls the grid connected inverter alone. The improved state following controller can reduce the impact of the switching moment of the controller on the voltage and current of the microgrid to the minimum value, and make the switching from grid connected to island operation smoother.

\subsection{Simulation experiment of switching from island mode to grid mode}

In order to improve the utilization of distributed power and the reliability of microgrid operation, microgrid is in the state of grid connected operation in most cases. Therefore, the ability of micro grid connected to the grid switching is also an important topic to be studied. For the micro grid model of master-slave structure established in this chapter, before grid connection and switching, the micro grid is in island operation mode. The main control source is controlled by virtual synchronous generator and the slave control source is controlled by constant power. After receiving the grid connection command and starting the pre-synchronization control, the main control source will adjust the output voltage and frequency under the interference of the presynchronization control signal until the voltage amplitude is equal to that of the large grid and the phase is the same. After receiving the closing signal, the PCC is closed. At the same time, the main control source is switched to constant power control. The slave control source keeps constant power control throughout the process. According to the simulation requirements, the simulation conditions are set, as shown in Table 1 below. 
Table 1. Simulation conditions for grid connection and switching of microgrid

\begin{tabular}{ccc}
\hline \hline Time & $0-0.2 \mathrm{~s}$ (Island) & $\begin{array}{c}0.2-0.5 \mathrm{~s} \text { (Pre-synchronization to grid } \\
\text { connection) }\end{array}$ \\
\hline DG1 reference power & $10 \mathrm{~kW}$ & $10 \mathrm{~kW}$ \\
DG2 reference power & $15 \mathrm{~kW}$ & $15 \mathrm{~kW}$ \\
Load 1 power & $20 \mathrm{~kW}$ & $20 \mathrm{~kW}$ \\
Load 2 power & $5 \mathrm{~kW}$ & $5 \mathrm{~kW}$ \\
\hline \hline
\end{tabular}

\subsection{Simulation experiment of switching from grid mode to island mode}

In the process of grid connected operation of microgrid, it is necessary to consider the situation of passive off grid caused by exceptional circumstances or active off grid due to system needs. Therefore, it is also important to switch smoothly in the process of off grid. For the off-grid process, an improved state following control is proposed. In order to verify its performance, in this study, two kinds of state following control are simulated and compared. As required, both microgrid models are set to the same operation status as shown in Table 2:

Table 2. Simulation conditions of microgrid off grid switching

\begin{tabular}{lcc}
\hline \hline \multicolumn{1}{c}{ Time } & $\begin{array}{c}0-2 \mathrm{~s} \text { (Grid connected } \\
\text { operation) }\end{array}$ & $0.2-0.5 \mathrm{~s}$ (Island operation) \\
\hline DG1 reference power & $10 \mathrm{~kW}$ & $10 \mathrm{~kW}$ \\
DG2 reference power & $15 \mathrm{~kW}$ & $15 \mathrm{~kW}$ \\
Load 1 power & $20 \mathrm{~kW}$ & $20 \mathrm{~kW}$ \\
Load 2 power & $5 \mathrm{~kW}$ & $5 \mathrm{~kW}$ \\
\hline
\end{tabular}

\section{Results and Discussion}

\subsection{Simulation results of switching from island mode to grid mode}

According to the simulation conditions in Table 1, the pre-synchronization control is started at $0.2 \mathrm{~s}$.

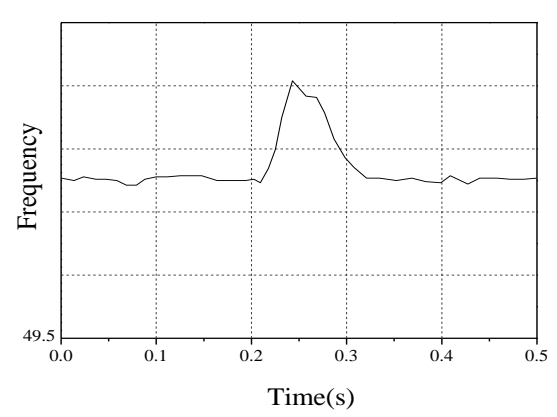

(a) Frequency variation of micro grid bus in the process of grid connection switching

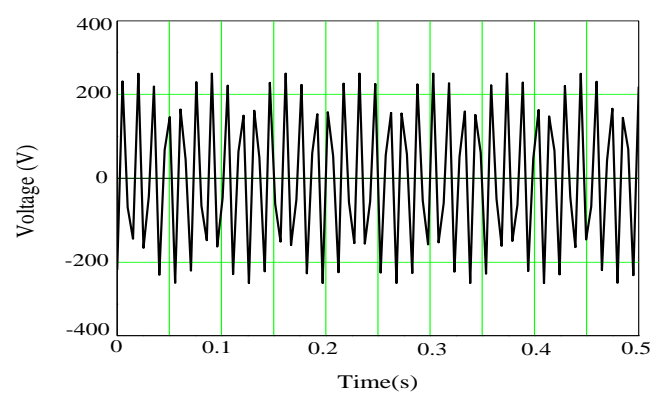

(c) Micro grid bus voltage in the process of grid connection switching
At the same time, in order to avoid the influence of power imbalance on the simulation results, the actual power output of the micro grid distributed generation is equal to the load power.

The simulation results are as follows:

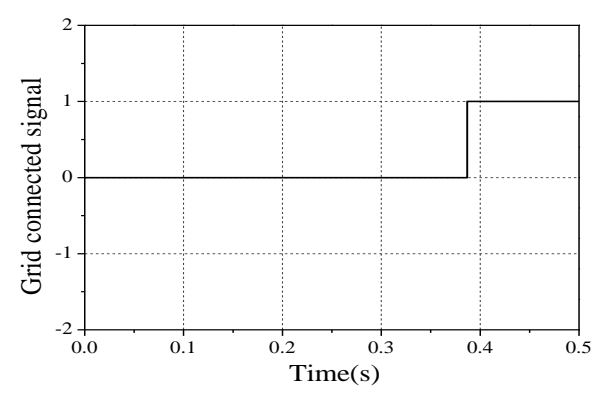

(b) Grid connection signal

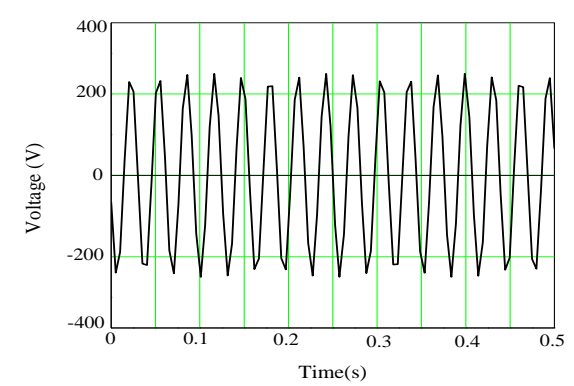

(d) Voltage (current) of microgrid at $0.2 \mathrm{~s}$ start-up pre-synchronization 


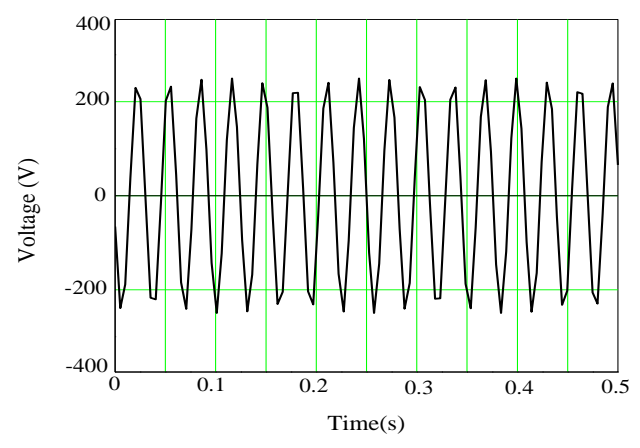

(e) Voltage (current) of microgrid at the end of pre-synchronization

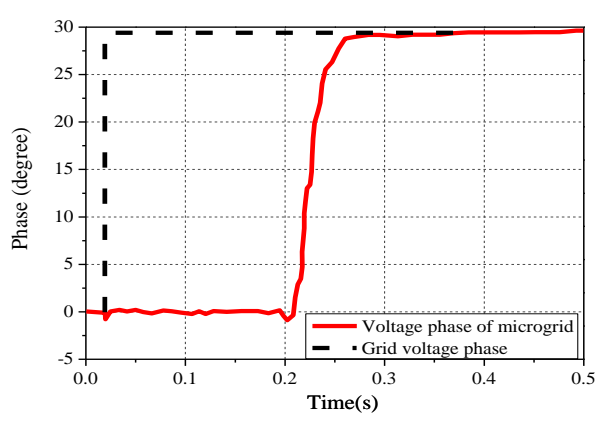

(f) The change of phase in pre-synchronization process

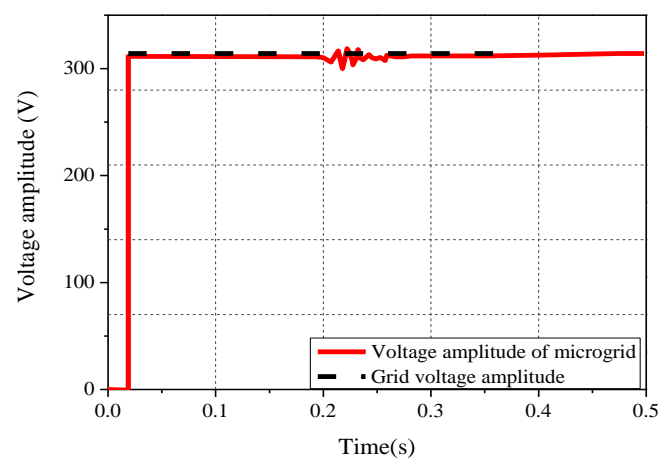

(g) The variation of voltage amplitude during pre-synchronization Figure 6: Simulation results of grid connection switching process

According to the simulation results in Figure 6 (f ) and (g), before sending the $0.2 \mathrm{~s}$ grid connection signal, the phase difference between the microgrid bus voltage and the grid voltage is nearly $30^{\circ}$, and the amplitude appeared same, so the phase presynchronization process is mainly analysed. According to the grid connection signal in Figure 6 (b), the pre-synchronization process ends at $0.3881 \mathrm{~s}$. During the process from $0.2 \mathrm{~s}$ when the presynchronization process starts to $0.3881 \mathrm{~s}$ when the pre-synchronization process ends, the frequency of microgrid bus shown in Figure 6 (a) will increase to catch up with the phase of large grid. Moreover, in the process of phase adjustment, the voltage frequency deviation is always within the allowable range. It can be seen from Figure 6 (d) and (e) that the voltage and current of the microgrid bus are very stable, basically without fluctuation, no matter at the start-up pre-synchronization control time of $0.2 \mathrm{~s}$ or at the end of pre-synchronization grid connection time of $0.3881 \mathrm{~s}$.

From the above simulation results, it can be seen that the pre-synchronization control strategy applied to the grid connection switching can effectively reduce the voltage and current impact during the grid connection.
At the same time, it results in the smooth switching of the grid connection process, which proves the effectiveness and feasibility of the presynchronization strategy proposed in this study.

\subsection{Simulation results of switching from grid mode to island mode}

From the simulation results in Figure 7 (a), it can be seen that the frequency variation of the off-grid process under the two-state following control is kept within the allowable range of the grid frequency deviation. The voltage of microgrid bus in the switching process of Figure 7 (b) is observed.

Compared with the voltage of microgrid in the off-grid time under the traditional state following control, the improved following control can keep the voltage stable before and after switching. Moreover, in the power changes shown in Figure 7 (c) and (d), the power impact of the microgrid under the traditional state following control at the time of off grid is also significantly greater than that under the improved state following control. 


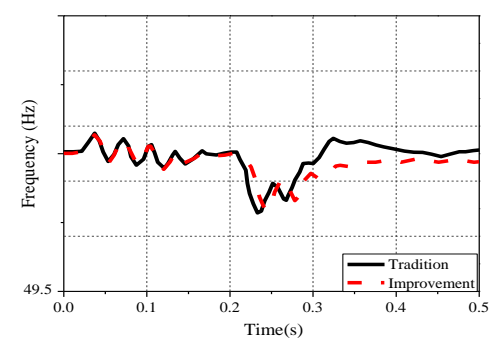

(a) Comparison between the frequency change of microgrid bus in the improved off grid switching process and that in the traditional off grid switching process

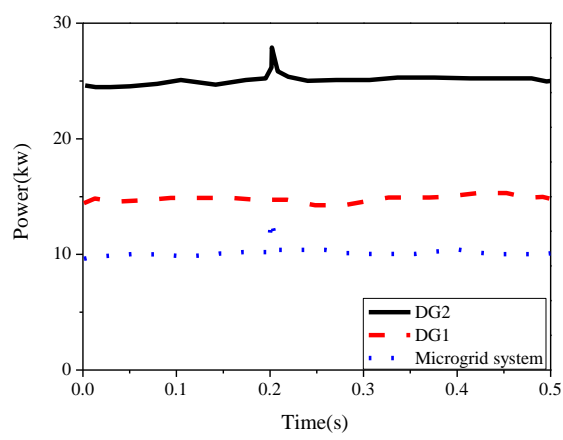

(c) Power change in the process of off grid switching after improvement

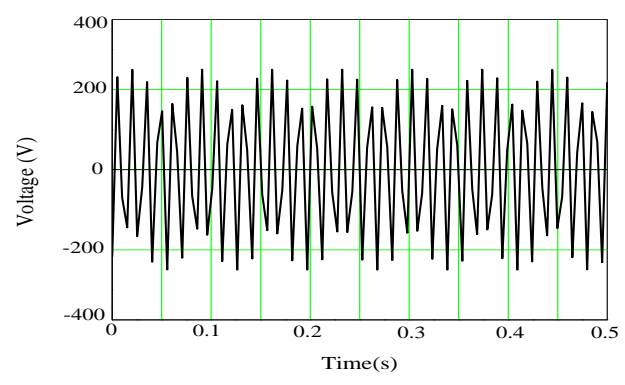

(b) Improved microgrid bus voltage in off grid switching process

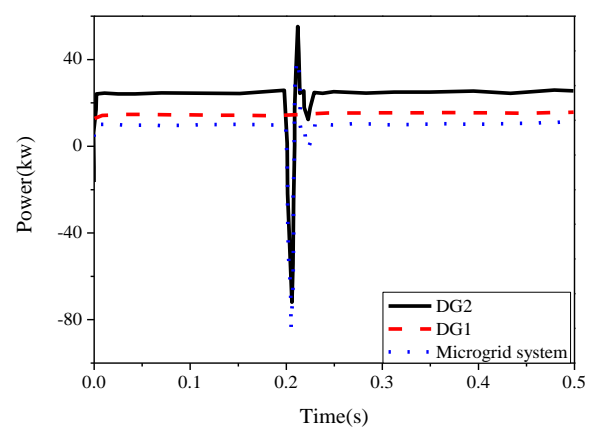

(d) Power change in traditional off grid switching process

Figure 7: Simulation results of off grid switching process

In conclusion, the simulation results show that compared with the traditional state following control, the improved method is more in line with the requirements of smooth switching from merging network to islanding, which proves that the improved method in this study is effective and benign.

\section{Conclusions}

In this study, a dual-mode parallel micro grid smooth switching control strategy is proposed by combining virtual synchronous generator control and constant power control. The pre-synchronization control strategy is designed to improve the traditional state following controller. According to the design of each control strategy, simulation experiments under different conditions are carried out.

The simulation results show that the effectiveness of each control strategy in this study not only realizes the stable operation of microgrid in island mode and grid connected mode, but also realizes the smooth transition in the mode switching process. In this study, each control strategy and smooth switching control strategy are mainly studied, ignoring the characteristics of different distributed generation and the influence of energy flow on the control strategy.
Therefore, it is necessary to improve the model further and introduce photovoltaic, storage battery and other models to make the simulation more practical. For the improved state following controller and its mechanism proposed in this study, further analysis and discussion should be carried out in combination with the automatic control principle, and appropriate simulation experiments should verify its effectiveness and stability.

\section{Acknowledgement}

Research on monitoring and energy management of distributed smart micro grid (Project No.: j18kb165)

\section{References}

[1] Wang X M, Liu H S, Chen Y F. Aerodynamic load control strategy of wind turbine in microgrid. Iop Conference, 2017, 100(1), pp. 012175.

[2] Safa A, Berkouk E L M, Messlem Y, et al. A robust control algorithm for a multifunctional grid tied inverter to enhance the power quality of a microgrid under unbalanced conditions. 2018, 100, pp. 253-264.

[3] Ghanbarian M M, Nayeripour M, Rajaei A, et al. Design and implementation of a new modified sliding mode controller for grid-connected 
inverter to controlling the voltage and frequency. 2016, 61, pp. 179-187.

[4] Abid Hossain Khan, Md Shafiqul Islam (2019). A Pctran-Based Investigation On The Effect Of Inadvertent Control Rod Withdrawal On The Thermal-Hydraulic Parameters Of A Vver-1200 Nuclear Power Reactor. Acta Mechanica Malaysia, 2(2): 32-38, DOI: 10.26480/amm.02.2019.32.38

[5] Zhou N C, Li C Y, Sun F Q, et al. Modelling and control of solid oxide fuel cell generation system in microgrid. Journal of Electrical Engineering, 2018, 68(6), pp. 405-414.

[6] Zhao H L, Yang Q Zeng H M. Multi-loop Virtual Synchronous Generator Control of Inverterbased DGs under Microgrid Dynamics. Iet Generation Transmission \& Distribution, 2016, 11(3), pp. 795-803.

[7] Ram Rohit Vannarth, K. Mallikharjuna Babu, P. Martin Jebraj (2019). Study On Sedimentation Stability Of Mahua And Simarouba Oil Based Magnetorheological Fluids. Acta Mechanica Malaysia, 2(2): 39-44, DOI: 10.26480/amm.02.2019.39.44

[8] Chen L, He H W, Zhu L, et al. Coordinated Control of SFCL and SMES for Transient Performance Improvement of Microgrid with Multiple DG Units. Canadian Journal of Electrical \& Computer Engi4neering, 2016, 39(2), pp. 158-167.
[9] M. Fayez, M. Mandor, M. El-Hadidy, F. Bendary (2019). Fuzzy Logic Based Dynamic Braking Scheme for Stabilization of Inter -Area. Acta Electronica Malaysia, 3(2): 16-22.

[10] Dong H N, Yuan S, Han Z J, et al. A Comprehensive Strategy for Power Quality Improvement of Multi-Inverter-Based Microgrid With Mixed Loads. IEEE Access, 2018, (99), pp. 1-1.

[11] Tu C, Yang Y, Xiao F, et al. The Output Side Power Quality Control Strategy for Microgrid Main Inverter under Nonlinear Load. Transactions of China Electrotechnical Society, 2018, 33(11), pp. 2486-2495.

[12] Hemad Heidari Jobaneh (2019). An Ultra-LowPower and Ultra-Low -Voltage $5 \mathrm{GHz}$ Low Noise Amplifier Design with Precise Calculation. Acta Electronica Malaysia, 3(2): 23-30.

[13] Mortaz E, Valenzuela J. Microgrid energy scheduling using storage from electric vehicles. 2017, 143, pp.554-562.

[14] Lezama F, Palominos J, Rodríguezgonzález A Y, et al. Agent-Based Microgrid Scheduling: An ICT Perspective. 2017, (1), pp. 1-17.

[15] Ghazala Nazir, Shaista Gul (2019). Comparative Study Of Mathematical Model Of Ebola Virus Disease Via Using Differential Transform Method And Variation Of Iteration Method. Matrix Science Mathematic, 3(1): 17-19. 